\title{
Addressing Sensitive Issues in Family Mediation: An Albanian Study of Mediator Perceptions
}

\author{
Dr. Klodiana Rafti \\ European University of Tirana, Albania \\ Dr. Sofjana Veliu \\ European University of Tirana, Albania
}

\begin{abstract}
Family mediation is proposed as an alternative approach to conflict resolution, whereby two or more parties to a dispute attempt by themselves, on a voluntary basis, to reach an agreement on the settlement of their dispute with the assistance of a mediator. The objective of this qualitative study was to explore the way mediators address salient issues in mediation, and how it may have an impact on resolving family disputes. The study focused on which are the mediators' perceptions with respect to a range of sensitive issues in mediation process; with regard to sensitive controversy how do mediators identify the needs of mandatory mediation; how mediators consider the inclusion of children in family mediation process; how mediators position themselves in issues such as child and spousal abuse situations in relation to mandatory mediation; and finally, what are the mediators perception with regard to issues linked to pathological behavior from the part of the disputants in relation to mandatory mediation. Consistent with a qualitative approach, semi-structured interviews were chosen to access the actual experience of mediators. The sampling frame was purposive in the study. Furthermore, the sample consisted of interviews with twenty mediators drawn from the Albanian National Chamber of Mediators. Regarding the debate whether mandatory mediation is one of these successful practices for solving family disputes in contrast to voluntary mediation, the findings suggest that most of mediators agree that mediation should only be on a voluntary basis. Most of them disagree with mandatory mediation, since it may have a negative impact on mediation, where possible manifested physical or psychological abuse is screened during mediation sessions. The findings also reveal that most of mediators are not in favor of mandatory mediation, since it may contribute to the failure of mediation, especially where dispute cases related to pathological behavior from the part of one of the disputants is identified during mediation. Furthermore, the decision on whether to include children or not in the mediation process is linked to context, expertise, professional experience from the part of the mediator, and other related factors. As opposed to including children in mediation, most of mediators in the study listed that children should not be included in the mediation process for the following reasons such as, the unwillingness of parents to include children in mediation, the age of the child, and the psychological/emotional state of children. In conclusion, the findings show that the way family mediators address sensitive issues in the field is not only related to mediator's competence of working methods, but it is also linked to nature of conflict, and disputants' individual characteristics. However, further research is needed to determine the effectiveness of family mediation in Albania with regard to models of practice, techniques, strategies, and salient issues in the field.
\end{abstract}

Keywords: family mediation, conflict, dispute resolution, sensitive issues, mandatory, child inclusion

\section{Introduction}

Family mediation is proposed as an alternative approach to conflict management in couples seeking the divorce, where a third, impartial person, named mediator operates to facilitate the dispute resolution processes. According to Lisa Parkinson (1997), family mediation is a process that allows the parties to handle their own problems, unlike the approach offered by the procedural system. In other words, family mediation is an alternative system created to regulate interpersonal disputes. 
Moreover, the purpose of this study is to explore the way mediators address salient issues in mediation, and how it may have an impact on resolving family disputes. The study focused on which are the mediators' perceptions with respect to a range of sensitive issues in mediation process; with regard to sensitive controversy how do mediators identify the needs of mandatory mediation; how mediators consider the inclusion of children in family mediation process; how mediators position themselves in issues such as child and spousal abuse situations in relation to mandatory mediation. This study will also look at what are the mediators perception with regard to issues linked to pathological behavior from the part of the disputants in relation to mandatory mediation.

With regard to process and practice of family mediation, this study has a great contribution in the family mediation field since it has received very little attention in general in the Albanian context. It should actually be the focus of research in the future to promote the field of family mediation to a more effective level of practice. Since there is lack of research regarding the way mediators address sensitive issues in the field, in the attempt to resolve family disputes, it is very important to generate specific data regarding mediation practice. This study may also contribute for further research within the field of family mediation regarding the mediators' working methods, including mediators' perceptions, beliefs, behaviors in relation to principles, models of practice, and mediators' roles/styles in Albania.

\section{Literature Review}

\subsection{Research in mediation}

Consistent with academic literature, various studies show that mediation ensures positive results for children. Furthermore, research conducted on parents who had experienced mediation showed that $58 \%$ of them believed that the mediation had helped them to protect the interests of children, while $37 \%$ thought that mediation had contributed to reaching agreements with regard to custody issues. The studies revealed that global mediation had been a tremendous help in addressing the problem of children, whereas family centered mediation focused on children, gave parents the chance to have contact with the younger son (McCarthy and Walker, 1996).

Moreover, Pearson and Thoennes (1988), in their extensive research in the US, found that mediation on issues relating to children represented an improvement over the judicial system, but could not solve all the problems during and after divorce. The researchers revealed a number of reasons to explain the fact that studies on mediation does not show significant and measurable benefits for the children. These included problems in establishing sufficiently sensitive issues, the period of time considered, and a number of other variables. Although mediation helped parents to reach decisions concerning children, and helped non-resident parents to maintain contact with them, however, the mediated agreements between the parents did not necessarily facilitated the psychological adjustment in children. The researchers concluded that the adaptation of children after a divorce depends on the interaction of many variables, including the quality of parenting, relationships between parents, family dynamics, and the environment surrounding the child, rather than the choice of parents to take part in mediation.

However, research shows that the involvement of children in mediation may produce positive effects in resolving family conflicts. Both in Britain and in other countries, it is shown that, even when the mediators support a policy that favors the direct involvement of children in mediation, only a small percentage of cases are actually involved. A Scottish study conducted by Garwood (1989) brought to light that although mediation services of Edinburgh would support a policy aimed at enhancing the participation of children, only $20 \%$ of cases were actually involved in mediation. From the mediators' point of view, the main reason for not engaging children in mediation sessions was their young age (average three and a half years). However, for parents, the most frequent reason was that they considered as unnecessary that children meet the mediator, because they felt able to talk to their children at home by themselves. Also, the study included controlled interviews with the children who had met with the mediator. Furthermore, the main modalities to involve children in mediation sessions consisted in meeting them separately without the parents. The sessions, mainly consisted of a discussion with the children, especially with the youngest ones.

The study revealed that most of the children reported that the goal for their involvement in mediation was not clear, and that for this reason they felt nervous and insecure. This seems to be the result of a lack of adequate information and explanations from the part of the mediators. Some of the children reported that they would prefer receiving a letter from the mediator. Several of them thought that the mediator should make decisions for them and for their family: two boys, aged 
nine and eleven years, thought that "the mediator would decide with whom they had to go to live with" (Garwood, 1989, p. 31).

Despite the uncertainty regarding their participation, almost all children considered very positive their own experience with the mediator. Saponseck (1991) commented on the lack of research conducted in the United States, and Britain about the involvement of children in mediation, and stressed out that it is important to conduct other large-scale studies, otherwise, it would be unwise to draw any conclusions with regard to the benefits of the involvement of children in mediation.

\section{Methodology}

\subsection{The purpose of the study}

The purpose of the study was to develop data on the experience of mediators in order to explore the way mediators address salient issues in the field, and how it may have an impact on resolving family disputes.

\subsection{Research questions of the study}

Which are the mediators' perceptions with respect to a range of sensitive issues in mediation process?

Associated question 1: With regard to sensitive controversy, how do mediators identify the needs of mandatory mediation?

Associated question 2: With regard to salient issues, how mediators consider the inclusion of children in family mediation process?

Associated question 3: With regard to sensitive matters, how mediators position themselves in issues such as child and spousal abuse situations in relation to mandatory mediation?

Associated question 4: What are the mediators perception with regard to issues linked to pathological behavior from the part of the disputants in relation to mandatory mediation?

\subsection{Research design}

\subsubsection{Methods}

A qualitative approach was chosen in order to answer the research questions as well as the associated research questions of the study. Furthermore, studies on qualitative research show that recently, researchers focus their attention on qualitative methods in addition to applying quantitative research methods (Denzin \& Lincoln, 2000).

Even in this study of family mediation, and taking into account a complex and a sensitive issue, such as the issue of divorce, the study aims to provide a detailed picture on the actual experience of mediators in order to explore the way mediators address salient issues in mediation process from mediators' own point of view by using a qualitative method design.

The reason of why the sampling of the study consists of mediators drawn from the National Chamber of Mediation, underlines the importance of the family mediation field in which the mediators face with divorce cases at their work settings on daily bases, so that the mediators represent of a valuable resource in this research, in the attempt to fulfill the main objectives of the study. With regard to the gender of the mediators, the study consists of eight (8) female mediators and twelve (12) male mediators. Interestingly, the study shows that gender does not have an impact on the effectiveness of mediation with regard to mediators' working methods.

\subsubsection{Instrumentation and data analysis}

Consistent with a qualitative approach, semi-structured interviews were chosen to access the actual experience of mediators. Furthermore, the sample consisted of semi-structured interviews with twenty mediators drawn from the Albanian National Chamber of Mediators. Considering the exploratory nature of the study, the goal of the semi-structured interviews was to allow the participants the freedom to express their views with regard to specific issues in the field, and to allow them express themselves through open dialogue resulting in a narrative form of communication. Also, this type of interview takes the form of self-report measures, which exposes each participant's held viewpoints by eliciting mediators' self-perceptions, roles, behaviors, and values about family mediation. 
Likewise, the purpose of these interviews was to discuss the mediators' perceptions on the effectiveness of family mediation with regard to sensitive issues, and permitted discussion about the effectiveness of mediation in resolving with conflict family issues dealing with divorce. Again, the interview questions attempted to elicit mediators' viewpoints in relation to sensitive matters, such as the issue of mandatory versus voluntary mediation, issues linked to pathological behavior from the part of the disputants in relation to mandatory mediation, the inclusion of children in mediation, and spousal/child abuse.

\subsubsection{Sample/Participants}

For the purpose of this study, a qualitative approach was chosen to access the actual experience of mediators in the attempt to generate more in depth answers from mediators who have a professional experience in family resolution conflicts. Furthermore, a crucial part of the present study is determining the sampling. It is important to highlight that before deciding what types of participants to include in a study, and what kind of information a researcher wants to collect, the main research questions of the study need to be identified and specified. Therefore, the main goal of the researcher should be clear from the start, by taking into account both the audience, and types of questions the researcher is going to include in the interviews. Furthermore, the sampling frame in the present study was purposive/judgment sampling, because it is selected based on the opinion of an expert. So, the study included mediators who have a professional experience as conflict resolution mediators. Likewise, in the judgment sampling, in the research, there is a selection of units to be sampled based on mediators' knowledge and professional judgement about family mediation, and working methods in the field.

Moreover, the sample consisted of interviews with twenty mediators drawn from the National Chamber of Mediators, that have been previously identified as working with conflict families and having an experience with family disputes matters.

In the study, (8) of them were male mediators and (12) of them were female mediators. The sample included only mediation professionals, in which most of them hold a Law University degree, mainly with backgrounds in family law, and very few of them included professionals with backgrounds in mental health etc., with years of practice ranging from one to seven years. Although, the study is primarily focused on conflict family disputes, mediators who partecipated in present study are also identified as having experiences on civil, commercial, and penal disputes. Furthermore, most of mediators hold a Law University degree, mainly with backgrounds in family law, and very few of them included professionals with backgrounds in mental health etc., with years of practice ranging from one to eight years.

\subsubsection{Procedure}

Furthermore, twenty interviews were conducted in the study, and their duration was for a period of six weeks (from May to July 2016). All participants in the interviews were informed about the procedures as well as the objectives of the study. They were explained their rights and, after being assured the ethical principles of confidentiality and secrecy, it was explained to them that they have the right to see the notes taken by the researcher, after the completion of the interviews. The duration of each interview lasted 35 to 40 minutes, and most of the interviews took place in the mediators' offices. Moreover, a note-taker was provided during the interviews, in order to gather information with regard to the most critical questions of the study.

The method of analysis used in this study is the management of data "manually" without using computer programs. Initially, the data collected were transcribed, and recorded manually through the interviews. Further, the researcher attempted to familiarize with all the data collected by reading and re-reading the transcripts, which served to get to know better the data and written comments prior to the left of the text. However, the transcription of interviews, keeping records and monitoring may provide a description for the study report but does not offer us further explanations. It is the competency of the researcher himself that gives meaning to data gathered by exploring and interpreting them.

\section{Results}

The purpose of this study was to develop data on the experience of mediators drawn from the Albanian National Chamber of Mediation in order to explore the way mediators address salient issues in mediation process from mediators' own point of view.

Furthermore, the views of the participants on the question of which are the mediators' perceptions with respect to a range of sensitive issues in mediation process is analyzed as the following. Also, four associated questions are emerged during the study in which mediators were asked how do they identify the needs of mandatory mediation. 
Moreover, the mediators were also asked how they consider the inclusion of children in family mediation process, and what they thought were the most crucial factors related with the children's divorce adaptation. Last but not least, to the respondents was posed the question of how they position themselves in issues such as child and spousal abuse situations with regard to mandatory mediation. Finally, the mediators were also asked what are their perception with regard to issues linked to pathological behavior from the part of the disputants in case of mandatory mediation.

Consider the following mediators' dilemma on whether mediation should be voluntary or mandatory in practice:

I believe that mediation should be on a voluntary basis only. Mediation is an alternative itself, but in order to become an alternative it has to be known, and it has to become a cultural choice, and for this we need time. So, at some point, I agree that mandatory mediation should be applied in specific cases of divorce.

Another important theme emerged during the study in which mandatory mediation may produce negative effects on the mediation process where manifested physical and psychological abuse is present during the process. Consider another lawyer mediator's comments in favor of voluntary mediation as opposed to mandatory mediation:

First, I do not think that mandatory mediation should be regulated by law in Albania, since not all the divorce cases are suitable to mediation. For example, the mandatory mediation is not a successful practice in cases where manifested physical or psychological violence, especially towards children or persons with disabilities is evident during the mediation process. Second, I think that all the disputing parties are free to enter the mediation, and they are also free to withdraw from the mediation process at any time they want to.

Consider another lawyer mediator's comments in favor of voluntary mediation as opposed to mandatory mediation where manifested physical or psychological violence is screened:

Since mediation is an alternative itself, and as such, it should be on a voluntary basis only. My professional experince as a mediator demonstrates that mandatory mediation is not the right alternative to resolve disputes when faced with spousal/child abuse situations. There are specific divorce cases which cannot be mediated. In this context, mandatory mediation is not the right solution. I strongly believe in the free will.

In addition, applying mandatory mediation in practice, could possibly damage or fail the mediation process itself, if divorce cases linked to pathological behavior from the part of one of the disputants is identified during the process. In this case, mandatory mediation would not result efficient in terms of achieving an agreement where one of the disputants may be previously diagnosed with a personality disorder or/and other pathological disorders as well. Consider the following mental health mediator's comments regarding the risks of the mandatory mediation in practice:

I think mandatory mediation may fail the process of mediation itself where pathological behavior is manifested during the mediation sessions. In such cases, I would stop the procedure, and suggest to the individual who displays symptoms of pathological behavior to meet a mental health expert. You can't pretend for the parties to assign an agreement, if one of them is previously diagnosed with narcissistic personality disorder, for example. That won't be reliable.

Similarly, consider another mediator's comments as the followings:

I believe that mandatory mediation could produce negative effects only, especially where cases of manifested pathological behavior is present. I am not a mental health expert myself, but eventhough disputant's pathological behavior is not present at first, again, manifested power imbalances issues emerge in mediation, where one of the disputants attempts to dominate the other. Instead, I am in favor of voluntary mediation.

With regard to mediation as voluntary or mandatory, consider the mediator's perceptions on viewing the mediation itself as staying in the middle of two:

I think that mediation should be on a voluntary basis only. Nevertheless, I think that some sort of mandated elements, depending on the context and the cases mediated, should be taken in consideration. I recommend a mandatory first session of mediation, because this may help the disputing party to get information about the mediation as a process. Then the process should be voluntary. In such conditions, the disputing parties are free to chose whether to enter the mediation or not. 
Similarly, another mediator expresses his views regarding voluntary versus mandatory mediation as staying in the middle of two:

Well, I am in favor of voluntary mediation, but it depends on the willingness of the mediators to inform their clientele about the nature, and the process of mediation as an alternative for family disputes. However, I suppose that some mandatory elements should be taken into account, and implemented as well, specifically in the first sessions of mediation.

Interestingly, one of the mediators expresses his direct views as being in favor of mandatory mediation as opposed to voluntary mediation, mainly linked to custody issues:

I am actually aware of the fact that mediation itself needs time to become a culture in our country, but I still believe that if mandatory mediation takes place, then it could positively contribute to the effectiveness of family dispute resolution in general, it mainly helps the parents solve custody issues, especially where their mutual interest are their children, and it contributes to the increase on the number of mediated cases. Not every judge reffers the cases of divorce to mediation, although the law provides for both types of mediation, voluntary and mandatory.

In addition, the mediators were also asked how they consider the inclusion of children in family mediation process, and what they thought were the most crucial factors related with the children's divorce adaptation.

Furthermore, important themes emerged during the study highlighting the relationship between mediators' professional background, styles, models of practice and the decision of the child inclusion in the mediation process. Consistent with the academic literature, the study shows that the mediators' perceptions on the controversy in favor or opposed to including children in mediation explain why some mediators who adopt the facilitative mediation disagree with the child inclusion in mediation. On the other hand, those mediators who adopt another kind of model (transformative) and who are particularly sensitive on child issues, are in favor of including children in mediation. The study shows that few of the mediators agree that the participation of the child in the mediation process could emotionally benefit the child and have a positive impact on the outcome. Consider the following comments of a mediator who is in favor of child inclusion in mediation:

I think that children have to be aware of what ia actually happening. They need to be informed of their parents' dispute and the divorce situation. I am quite sure that children experience feelings of confusion during the stages of divorce, and they should be helped by getting the right information with regard to their parents' agreement.

Consider another example where the mediator's views on the child inclusion in mediation coincides with her professional background as a psychologist:

Since the phenomenon of divorce produces in children more the experience of feelings than the thoughts, I think that children's feelings and needs should be listened and they should have a voice in the process. By participating in the process, children can be helped adjust emotionally, and have an awareness of how to cope with conflict.

As opposed to including children in mediation, most of mediators in the study agree that children should not be included in the mediation process for the following reasons listed below. Furthermore, major themes emerged during the study, such as the unwillingness of parents to include children in mediation, the age of the child, the psychological/emotional state of children, the appearance of symptoms of stress to the child created as possible manipulation from the part of the parents. Taking into account mediators' perceptions on not including children in mediation, most of them agree that these salient factors, may cause the failure of the mediation process itself. Consider the following mediators'comments:

I think there are specific circumstances in which children should not participate in the mediation process. If parents do not wish to bring their children in mediation, and they certaintly want to resolve their problems by their own without involving them, then there is nothing to do. If children are involved in the process against their parents will, then this wouldn't work. Instead, they may feel not free to talk about.

Furthermore, consider another mediator's perceptions who does not support the idea of including children in mediation as linked to the unappropriate age of the child to deal with legal issues:

I do not think that children should be included in mediation, since a child is not prepared to understand the dynamics of law, and talk about legal issues. Instead, a child needs more of psychological support in order to face with the consequences of divorce. 
Similarly, another mediator does not support the idea of including children in mediation because of their age:

I do not think that children should participate in mediation sessions. They are not psychologically matured enough, and also they are not emotionally prepared to face with custody related issues. Besides, mediators should be trained enough to include children in mediation.

Furthermore, family and social factors may contribute to the non iclusion of children in mediation as one of the mediators explains as the followings:

I think that including children in mediation could not work, since children might be manipulated or influenced by their parents, or by one of their parent's family of origin to such an extent that it could not lead to the effectiveness of resolving family conflicts.

Interestingly, another mediator expresses his ideas with regard to the inclusion of children in mediation as staying in the middle of two: his willingness to include children in mediation, and his sceptical views on the benefits that mediation could produce for children.

From the one hand, I think that children could benefit from mediation, since they face with social as well as psychological post divorce consequences, and this could help them a lot. On the other hand, I doubt that mediators practice it, since we haven't yet developed a culture and practice of family mediation in Albania.

Similarly, another mediator expresses his views with regard to the inclusion of children in mediation as staying in the middle of two:

I think that children should partecipate in mediation up to ten years of age in joint sessions with their parents. In these cases, I suggest that a follow up after mediation should be done, even if the case has not settled. However, I barely believe that the partecipation of children in mediation could produce any effects in the attempt to negotiate.

In addition, another mediator suggests that the unappropriate age of the child does not guarantee reliability in terms of parental custody due to child immaturity. Consider the following example:

I think that the age of the child is a crucial factor, which in my views it strongly determines the effectiveness of mediation. Even if the child is more than ten years old, he or she is unable to discuss custody issues, and this may negatively contribute to the creation of confusion in parents by not reaching an agreement at the end of the process.

Moreover, the emotional/psychological state of the child due to parental disputes linked to the divorce, is another crucial factor which does not support the idea of including children in mediation. Consider the following comments:

I believe that a child who is feeling anxious and stressed due to factors related to divorce, and he is feeling anxious about talking to a mediatior, is not able to get focused on custody related issues.

Furthermore, consider another mediator's perceptions in relation to cases when children might be manipulated by their parents to such an extent as to cause significant stress and anxiety to their children:

From my own experience as a mediator, there are parents in which behave in a very egocentric way by focusing themselves in their positions rather than focusing on the best interest of the child. They are not able to differentiate their own needs from their children's needs and wishes. I do not think that in such cases, children may be included in the mediation process.

\section{Discussion}

Furthermore, the views of the participants on the question of which are the mediators' perceptions with respect to a range of sensitive issues in mediation process is discussed as the following. Also, four associated questions are emerged during the study in which mediators were asked how do they identify the needs of mandatory mediation.

Moreover, the mediators were also asked how they consider the inclusion of children in family mediation process, and what they thought were the most crucial factors related with the children's divorce adaptation. Last but not least, to the respondents was posed the question of how they position themselves in issues such as child and spousal abuse situations with regard to mandatory mediation. Finally, the mediators were also asked what are their perception with regard to issues linked to pathological behavior from the part of the disputants in case of mandatory mediation. 
It is interesting to consider the experience of mandatory mediation in countries like New Zealand, and the US, in order to see to what extent it confirms the theory, widely accepted, that the mandatory mediation would be ineffective, or would result in an unacceptable pressure on disputants. Mandatory mediation in the United States is mostly concerned with children-related issues. The court-ordered mediation to Los Angeles Conciliation Court is been considered as satisfactory and efficient, (from an economic standpoint) in defining child custody and visitation disputes. So, in 1981, the California puts a law in which, it requires to the divorcing parents to enter the mediation sessions before going to the court. Furthermore, the mandatory mediation was perceived as a justified attempt to resolve the conflicts on children. Its application in California, created lots of controversy (Grillo, 1991; Rosenberg, 1992). The researchers sought to determine how parents were experiencing the process, and if they felt satisfied with the outcome of mediation.

In 1992, in an important study on mediation, there was obtained information from a sample of participants equal to $83 \%$ of all mediation sessions (1.700) conducted in a two-week period. The cases addressed in mediation included high rates of violence domestic, child abuse and substance abuse. The mediators reported that $79 \%$ of the sessions dealt with difficult issues, and $71 \%$ dealt with high levels of emotional distress. Despite these problems, more than $80 \%$ of parents perceived the mediation sessions as positive. Moreover, the participants reported of not being pressed by the mediator, whereas the mediators determined as productive as $76 \%$ of the mediation sessions.

American researchers revealed strong public support for the mandatory mediation with regard to those who had participated in the study. At least $85 \%$ of those who had reached the agreements were in favor of mandatory mediation, and still $62 \%$ of those who had not reached agreements reported that mandatory mediation is probably necessary to resolve family disputes.

Coming to the actual study on family mediation, as regards whether mandatory family mediation, in its mitigated form or not, is one of these successful practices in Albania, the law provides for both types of mediation, voluntary and mandatory. Voluntary mediation can be undertaken by the disputing parties at any time and/or stage, regardless of whether a court proceeding has already started (art. 2.1). However, once a court proceeding is initiated, the judge must orient the parties towards mediation, especially for family law disputes and those where interests of children are at stake. The judge must refer to mediation also those cases where a mandatory reconciliation meeting is provided for under the Albanian Family Code (art. 4). This serves to confirm that reconciliation and mediation are two activities separate and independent from each other.

Regarding the debate whether mandatory mediation is one of these successful practices for solving family disputes in contrast to voluntary mediation, the findings suggest that most of the mediators agree that mediation should only be on a voluntary basis. Most of them disagree with mandatory mediation, since it may have a negative impact on mediation, where possible manifested physical or psychological abuse is screened during the mediation sessions.

The study also shows that most of the mediators are not in favor of mandatory mediation, since it may contribute to the failure of mediation, especially where dispute cases related to pathological behavior (for ex., personality disorders) from the part of one of the disputants is identified during mediation.

Few of the mediators view mediation as staying in the middle of two, and agree that although mediation is an alternative itself, mandatory mediation should be applied in specific cases of divorce, since mediation has not become a cultural choice yet from the parts of the disputants.

Consistent with the academic literature, research shows that the involvement of children in mediation may produce positive effects in resolving family conflicts. Both in Britain and in other countries, it is shown that, even when the mediators support a policy that favors the direct involvement of children in mediation, only a small percentage of cases are actually involved. A Scottish study conducted by Garwood (1989) brought to light that although the mediation services of Edinburgh would support a policy aimed at enhancing the participation of children, only $20 \%$ of cases were actually involved i mediation. Despite the uncertainty regarding their participation, almost all children considered very positive their own experience with the mediator.

Coming to our study, the decision on whether to include children or not in the mediation process is linked to the context, the expertise, and the professional experience from the part of the mediator. In addition, taking into account of what is "the best interest of the child" also is linked to the style and the model of practice from the part of the mediator. As Wallerstein \& Kelly (1980) suggest, mediation is a process in which the disputing parties are invited to negotiate and therefore settle 
an agreement for their best interest without involving children in their disputes. Similarly, the findings show that mediators who adopt the facilitative approach, are less likely to include children in the mediation, suggesting that the disputing parties are invited to settle an agreement for their best interest without the involvement of children. On the other hand, Lansky (1996) points out that mediators from a mental health background are more likely than lawyer mediators to include children in mediation. Finally, the desicion on whether to include children ot not in the mediation is culturally determined. According to Saposneck (1991), not all the cultures support children's rights to enter a mediation session, since in some countries the children's needs and wishes are satisfied by their own parents, whereas other cultures support the autonomy of the child to decide by his/her own.

Furthermore, the study also highlights the importance of the relationship between mediators' professional background, styles, models of practice and the decision of the child inclusion in the mediation process. Consistent with the academic literature, the study shows that the mediators' perceptions on the controversy in favor or opposed to including children in mediation, explains why some mediators who adopt the facilitative mediation disagree with the child inclusion in mediation. On the other hand, those mediators who adopt another kind of model, and who are particularly sensitive on child issues, are in favor of including children in mediation. The study shows that only two (2) out of twenty (20) of the mediators agree that the participation of the child in the mediation process could emotionally benefit the child and have a positive impact on the outcome.

As opposed to including children in mediation, most of the mediators in the study listed that children should not be included in the mediation process for the following reasons such as, the unwillingness of parents to include children in mediation, the age of the child, the psychological/emotional state of children, the appearance of symptoms of stress to the child created as possible manipulation from the part of the parents. Taking into account mediators' perceptions on not including children in mediation, most of them agree that these salient factors, may cause the failure of the mediation process itself. Specifically, most of the mediators emphasized that the unappropriate age of the child does not guarantee the effectiveness of mediation in terms of parental custody.

Finally, when it comes to salient issues in mediation, the findings suggest that with regard to disputes' characteristics in the attempt to solve salient matters, most of the participants identified as crucial in their work the children focused issues, power imbalances issues, conflict escalation of the disputes, and parties' cooperation in reaching an agreement.

\section{Conclusions}

The findings of the study provide an empirical evidence of family mediation practice in Albania analyzing the factors and features affecting mediation in terms of the way mediators address problematic issues in mediation.

As regards to sensitive issues, most of mediators disagree with mandatory mediation, since it may have a negative impact on mediation, where possible manifested physical/psychological abuse is screened, and possible manifested pathological behavior is present. Only few of them view mediation as staying in the middle of two, and agree that although mediation is an alternative itself, mandatory mediation should be applied in specific cases of divorce, since mediation has not become a cultural choice yet from the parts of the disputants.

Furthermore, the findings show that the decision on whether to include children or not in the mediation process is linked to the context, expertise, and professional experience from the part of the mediator. In addition, taking into account of what is "the best interest of the child" is also linked to the style and the model of practice from the part of the mediator. The study shows that only few of the mediators agree that the participation of the child in the mediation process could emotionally benefit the child and have a positive impact on the outcome. As a result, this brings about the necessity for the mediators support additional training before they involve children in mediation.

However, it is impossible to conceive a search that can measure the intrinsic value of the mediation process, because it is too complex and includes too many factors.

Interestingly, during the eighties, the researchers evaluated the mediation especially in terms of percentages in reaching agreements, and the disputants levels of satisfaction. Nowadays, there is more attention to the outcomes in the long term, to different aspects of the role of the mediator, and to models of practice currently in-use. Moreover, some researchers argue that mediation should consider long-term sessions for disputants, rather than focusing on the resolution of the dispute in the short term (Walker and Hornick, 1996). Is it reasonable, however, to expect the mediators resolve the conflicts that 
accompany the double failure, address the needs of children, attempting to resolve the financial and property disputes, and also individually assist disputants in their long-term psychological recovery? If one agrees to all of these objectives, then, this produces important implications for the preparation of the mediators, and the level of funding needed. From the other hand, may the mediation outcomes justify the continued commitment to its further development?

In conclusion, in terms of mediation in general, and family mediation in particular, trying to provide unifying principles for a multitude of countries is, at the very least, challenging. Having different perspectives from different realities may help in identifying practices that have in common the potential for being successful, by developing clarified practice models, and new strategies which may enhance the efficiency of mediation, despite the nature of conflict, individuals' characteristics, and problems in general. However, having a good and clear regulatory framework is the first step to creating and developing a culture and practice of family mediation. Finally, having a clear idea in creating new strategies of practice, and training qualifications for mediators to specifically enable them address a variety of salient issues in the attempt to solve family conflicts, requires not only the legislative willingness to change, but also the implementation of these mediation standards of practice in the field.

Also, a more clarified classification of the models/eclectic approaches to practice is needed, when taking into account nature of conflict, parties' individual traits, power imbalances issues, parties' communication/negotiation skill level, social/cultural aspects etc, and also when present sensitive issues need to be appropriately addressed in practice from the part of the mediators. Consequently, having a more sophisticated strategy for resolving family disputes, this could enhance the efficiency of positive outcomes in mediation.

\section{References}

[1] Davis, G \& Lees, P. (1981), A study of conciliation: Its impact on legal aid costs and place in the resolution of disputes arising out of divorce, University of Bristol, Department of Social Administration.

[2] Garwood, F. (1989), Children in conciliation, Edinburgh, Scottish Association of Family Conciliation Services.

[3] Grillo, T. (1991), The Mediation Alternative: Process Danger for Women, "Yale Law Journal", vol. 100, n. 6, p. 1545-1610.

[4] McCarthy, P \& Walker, J. (1996), Evaluating the longer term impact of family mediation. In

[5] Parkinson, L., (2003), L'esperienza inglese dei servizi di Mediazione Familiare, in Scabini, E.

[6] Parkinson, L., (1997), La mediazione familiare: modelli e strategie operative, Erikson, Londra, 1997;

[7] Pearson, J \& Thoennes, N. (1984), A preliminary portrait of client reactions to three court mediation programs, "Mediation Quarterly", vol. 3, p. 21-40.

[8] Pearson, J \& Thoennes, N. (1988), Divorce mediation: Research results. In Folberg, J \& Milne, A, Divorce mediation: Theory and practice, New York, Guilford Press.

[9] Rosenberg, J. (1992), In defence of mediation, "Family and Conciliation Courts Review", vol. 30, n. 4, p. 422476.

[10] Saponseck, D. (1991), The value of children in mediation: A cross-cultural perspective, "Mediation Quarterly", vol. 8, n. 4, p. 325-342.

[11] Walker, J \& Hornick, J. (1996), Communication in marriage and divorce, The BT Forum.

[12] Wallerstein, J \& Kelly, J. (1980), Surviving the break-up: How children and parents cope with divorce, London, Mclntyre. 